\title{
An evaluation of echography in the diagnosis of pancreatic disease
}

\author{
G. FOnTANA, L. BOLONDI, M. CONTI, G. PliCCHI, L. GUllo, \\ G. C. CALETTI, AND G. LABO \\ From the Medical Clinic II of the University of Bologna and Bioengineering Department of \\ Sant'Orsola Hospital, Bologna, Italy
}

SUMMARY In the present study an attempt to evaluate the efficacy of echography as a diagnostic tool has been made. A total of 52 patients (chronic pancreatitis (42); pancreatic cysts (three); and carcinoma of the pancreas (seven)) were studied and the results compared with those from other diagnostic techniques. In $65.7 \%$ of chronic pancreatitis patients, and in all cases of carcinoma of the pancreas, echography provided evidence of pancreatic abnormality but in no case could an unambiguous diagnosis of the disease be made. However, in all cases of pancreatic cyst, echography gave precise and unequivocal diagnostic information. There was good agreement between the echographic picture and surgical findings. Cholangiography and duodenography indicated duodenal and choledochal compression in a high proportion of cases in which echography revealed enlargement of the head of the pancreas. It is concluded that echography is a simple, safe, and valuable addition to the techniques available for studying the pancreas.

Echography is a fairly recent addition to the methods available for the diagnosis of pancreatic disease and as little work has been reported in this field its diagnostic value has yet to be fully evaluated.

The normal pancreas is not always well visualized by echography and it is often difficult to distinguish from the surrounding structure; on the other hand, the diseased pancreas, which is usually enlarged and structurally altered, gives a well-defined picture which may be of great assistance in diagnosis (Engelhardt and Blauenstein, 1970; Filly and Freimanis, 1970; Templeton and Stuber, 1971; Weill et al., 1971; Stuber et al., 1972). In chronic pancreatitis, the characteristic feature of the echogram is total or partial enlargement of the gland and areas of non-homogeneous structure consisting of low echo zones alternating irregularly with high echo zones which may involve the entire pancreas or be confined to certain sections (Filly and Freimanis, 1970). In carcinoma of the pancreas echography reveals enlargement generally confined to one section of the gland, fairly uniform structure, and irregular outlines (Rettenmaier, 1975; Weill et al., 1975). However, it is in the diagnosis of pancreatic cysts that echography gives the clearest picture

Received for publication 9 January 1976 showing up transonic areas (typical of fluid collection) which appear as zones completely devoid of echos (Leopold, 1972).

In the present study, we describe our experience with this technique in disorders of the pancreas, attempting to assess its value as a diagnostic tool and comparing the results with those obtained from other diagnostic techniques.

\section{Method}

A total of 52 patients were investigated comprising 42 with chronic pancreatitis, three with pancreatic cysts, and seven with cancer of the pancreas. Diagnosis of chronic pancreatitis was firmly established by clinical, biological, and radiological data and later confirmed at surgery in 20 cases. All cases of cysts have since undergone surgical treatment and all cases of carcinoma were confirmed at surgery. Fifteen controls with no clinical, radiological, or functional evidence of pancreatic disease were also studied.

Echography in B scan was carried out in all cases (and repeated in some) after a 12 hour period of fasting and with the patient in the supine position. Routinely, a series of transverse scans were carried out at $1-2 \mathrm{~cm}$ intervals, beginning at the xiphoid process and followed by a series of sagittal scans 
extending left and right from the midline. In some cases, oblique scans were also taken.

Given the oblique position of the gland, only a portion of it is visible on transverse sectionsusually the head as it is the thickest part. The tail is generally hidden by the stomach or by the splenic flexure of the colon in echograms taken in the supine position (gas completely obstructs ultrasonic transmission), but it is well visualized in sagittal sections taken in the prone position at 4,6 , and $8 \mathrm{~cm}$ left of the midline. It is almost always visible anterior to the upper part of the left kidney.

In order to obtain more information on the morphology, the relationship and the structure of the various internal organs it is necessary to take several scans at different gains for each section. The gain allows for amplification of the echoes (of the various amplitudes, depending on the variations in acoustic impedance of the medium being examined), which are reproduced on the screen when above a certain level. Using low gain settings, echograms poor in echoes are produced. Only those corresponding to major differences in impedance-such as the passage from liquid to solid tissue and the boundaries between contiguous organs-have sufficient amplitude to be reproduced on the screen, visualizing the outlines of the internal organs (morphological gain). By increasing the gain the internal structure of the organs becomes evident (structural gain).

It is useful to repeat the same scan at a given gain setting in order to eliminate false echoes which sometimes occur, due to imprecision when the transducer on the surface of the skin is moved manually. Only the echograms faithfully reproduced in successive scans can be taken into consideration for interpretation.
In order to identify the pancreatic region with certainty, it is indispensable to have an anatomical point of reference for each section (liver, kidneys, vertebral column, vena cava, aorta). It is of particular importance to visualize the structure behind the organ being examined to be certain that it has been scanned in entirety. The screen is divided into square centimetres enabling exact measurements to be taken from the echogram. Echograms can also be photographed by the auxiliary Polaroid camera.

A Toshiba Sondayergraph and Picker Echoview were used in this study.

\section{Results}

Only in five of the normal subjects was there a picture clearly attributable to the pancreas. Transversally, it has a curved appearance with the concave edge towards the posterior wall (Fig. 1) and in sagittal sections, midline and left and right of the midline, it appears as a flattened oval positioned in the angle between the posterior edge of the liver and the aorta or the inferior vena cava (Fig. 2).

\section{CHRONIC PANCREATITIS}

In seven cases it was impossible, even after repeated attempts, to obtain a clear echogram because of the presence of air in the stomach and intestine and in $\mathbf{1 2}$ cases we were unable to detect any alteration in either the size or the structure of the pancreas. However, in the remaining 23 cases, pictures clearly attributable to a pathological condition of the pancreas were obtained. The pancreas was enlarged to a greater or lesser extent in all these cases; in 10 only the head was involved (Fig. 3) and in 13 the body (Fig. 4) and/or tail were also involved. An important

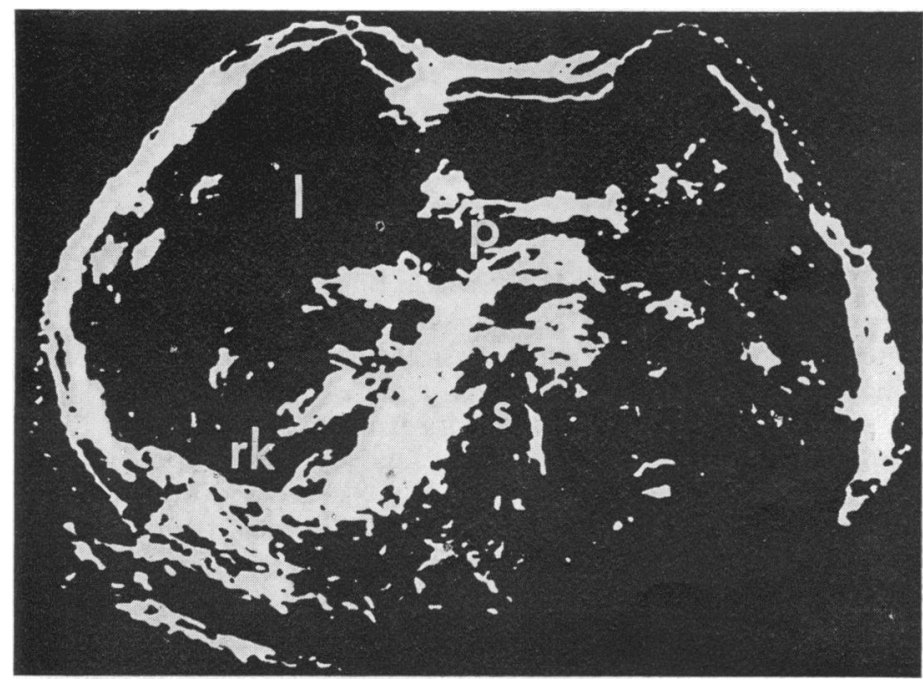

Fig. 1 Control. Transverse section 4 $\mathrm{cm}$ from the xiphoid process. Normal pancreas. $\mathrm{p}=$ pancreas, $\mathrm{l}=$ liver, $\mathrm{rk}=$ right kidney, $\mathrm{s}=$ spine. 


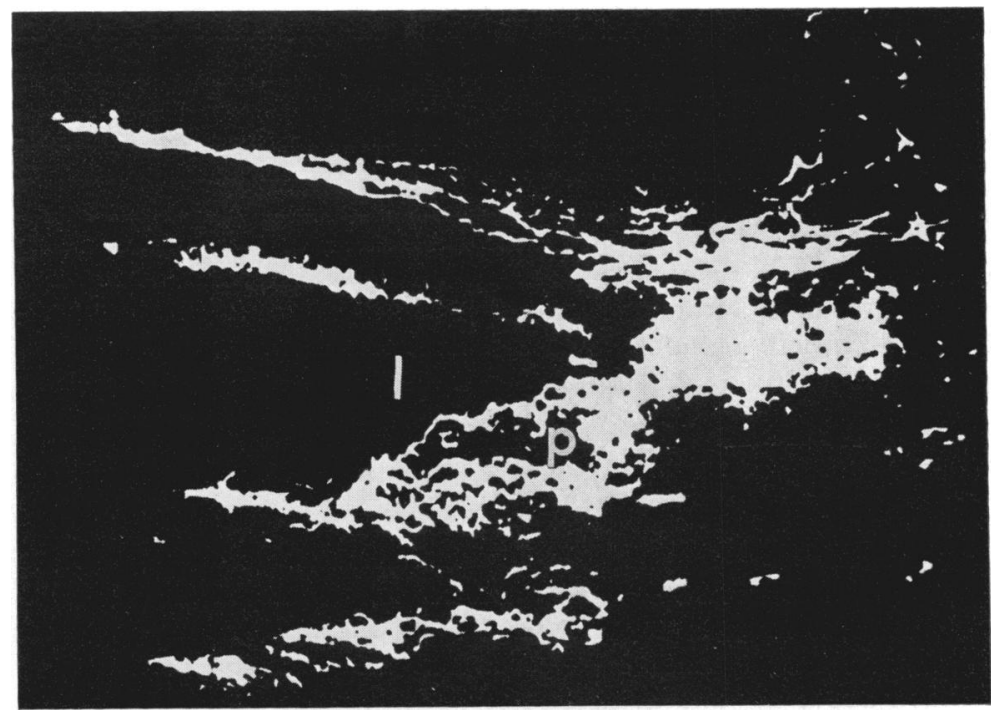

Fig. 2 Control. Midline longitudinal section. Normal pancreas.

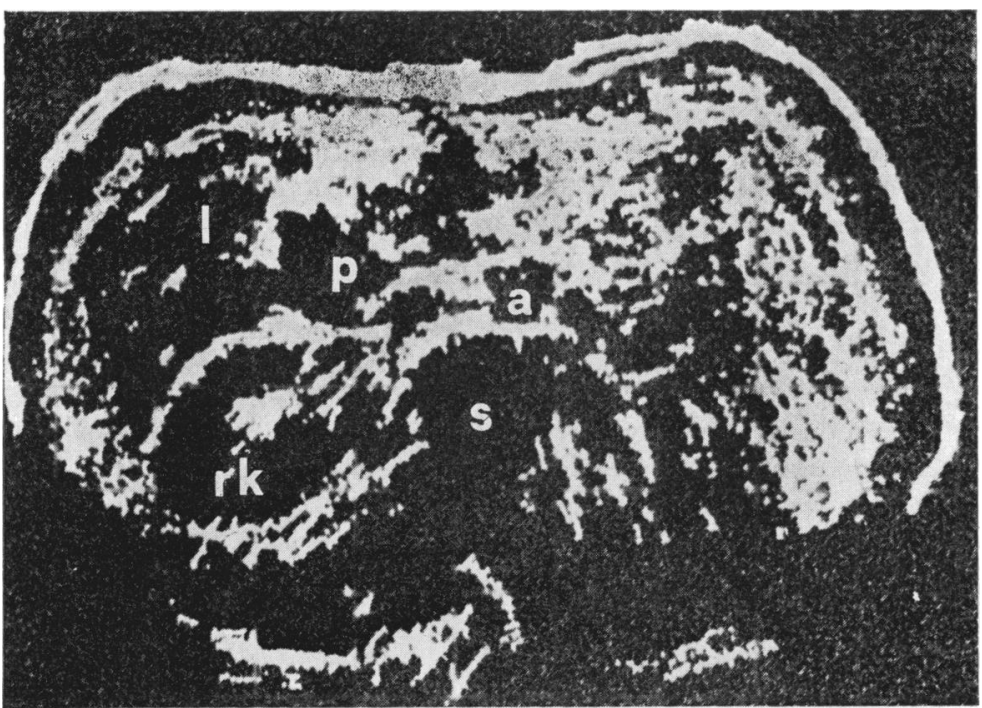

Fig. 3 Chronic calcifying pancreatitis. Transverse section $6 \mathrm{~cm}$ from the xiphoid process. $A$ well-delineated homogeneous area can be seen anterior to the right kidney and the vena cava, indicating enlargement of the head of the pancreas. $a=$ aorta.

point is that in all these cases the pancreas was visualized on the echogram as an area sharply delineated by the surrounding structures and this was perfectly reproducible even at a later date.

The structure of the pancreas was remarkably uniform (Figs. 3 and 4 ) in 10 cases but in the remainder randomly distributed multiple echoes appeared even at relatively low gain settings. In two cases a sharply defined transonic area characteristic of a cyst was seen in the head of the pancreas.

In cases with positive findings, clearer echographic pictures were obtained from transverse scans in four cases and from longitudinal scans in two cases. In the remaining 17 , both sections gave equally clear indications. However, in only 14 cases were the two sections in perfect agreement regarding the quantitative increase in size and the structural characteristics.

Comparison of data from echography with those from cholangiography and duodenography showed that, in the 23 cases with enlargement of the head of the pancreas, sign of compression of the duodenum and choledochus were present to a much greater degree than in the other cases ${ }^{1}$ (Table). Scintillo-

1 Details of these cases are obtainable from Professor G. Fontana. 


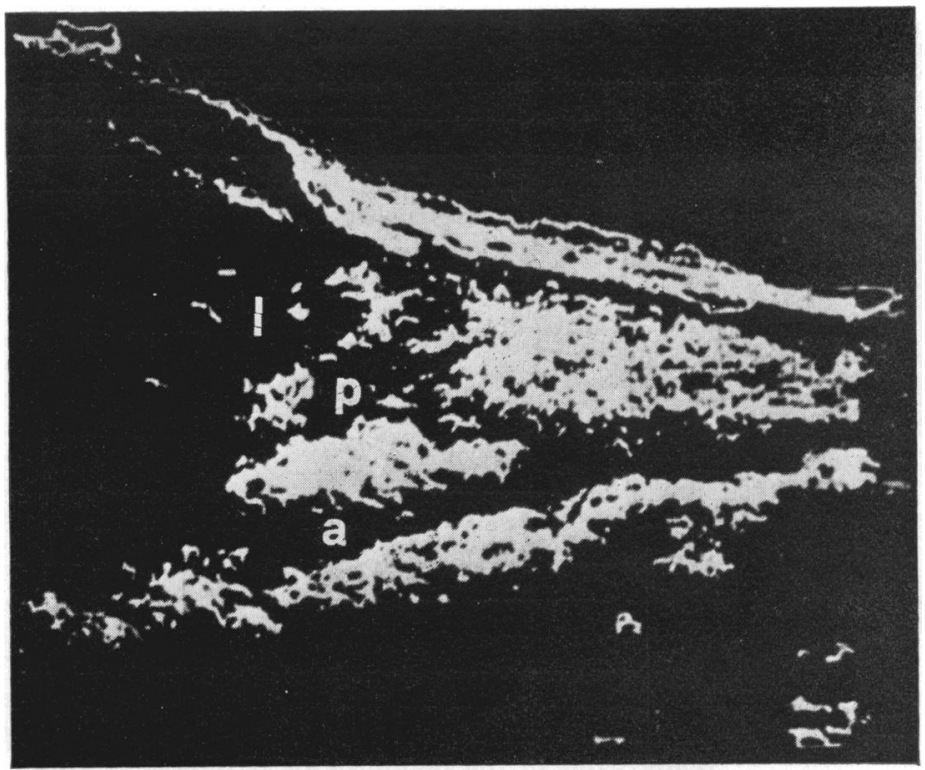

Fig. 4 Recurrent chronic pancreatitis. Longitudinal section $1 \mathrm{~cm}$ left of the midline. The enlarged pancreas is clearly visible between the liver and the aorta.

graphy was positive-that is, general or partial uptake defects were seen-in all except one case where echography was positive, and in all cases where echography was negative.

Good agreement was found between echographic data and surgical findings. In 11 cases showing marked enlargement of the head of the pancreas the extent of the increase was found to be identical when comparing measurements taken from the echograms and those taken during operation. In three cases the increase in size found at surgery was less than predicted from the echogram. In one of the two cases in which cyst of the head of the pancreas was also visualized, surgery provided confirmation.

Six of the 12 cases found to be negative at echography also underwent surgery; chronic pancreatitis was confirmed histologically but was unrelated to any change in the size of the organ.

\section{PANCREATIC CYSTS}

In addition to the cases of pseudocyst mentioned above, we had occasion to investigate three further cases admitted to hospital with a large abdominal mass of suspected pancreatic origin.

The first, a 16 year old girl with chronic calcifying pancreatitis, had developed a mass in the left hypochondrium. Endoscopic retrograde pancreatography and selective coeliac and superior mesenteric arteriography were unable to provide a conclusive diagnosis. Echography demonstrated the presence of a transonic zone completely devoid of echoes, even at very high gain, characteristic of pseudocyst formation (Fig. 5). Surgery confirmed the presence of a pseudocyst in the tail of the pancreas corresponding exactly in shape, size, and position with the echographic picture.

The second case (Fig. 6), a 53 year old man, presented with severe epigastric pain, elevated temperature, hyperamylasaemia and a mass in the epigastric region which appeared shortly after cholecystectomy for empyema of the gallbladder. A transverse echogram indicated the presence of a large elongated transonic zone extending from one hypochondrium to the other in the prevertebral region. Surgery confirmed the presence of a collection of liquid in the lesser sac of the peritoneum accompanied by liquefying necrosis of a large part of the pancreatic tissue.

The third case, a 34 year old man, presented with pain and a feeling of tension in the left hypo-

\begin{tabular}{llll}
\hline Echographic findings & \multicolumn{2}{l}{ Radiological findings } & \\
\cline { 2 - 4 } & Calcification (\%) & Alteration of duodenum (\%) & Alteration of choledochus (\%) \\
\hline Enlargement of head of pancreas (23 cases) & $65 \cdot 2$ & $71 \cdot 4$ & $81 \cdot 8$ \\
Negative echogram (12 cases) & $25 \cdot 0$ & $18 \cdot 2$ & $9 \cdot 0$ \\
\hline
\end{tabular}

Table Comparison of radiological and echographic findings in cases of chronic pancreatitis 


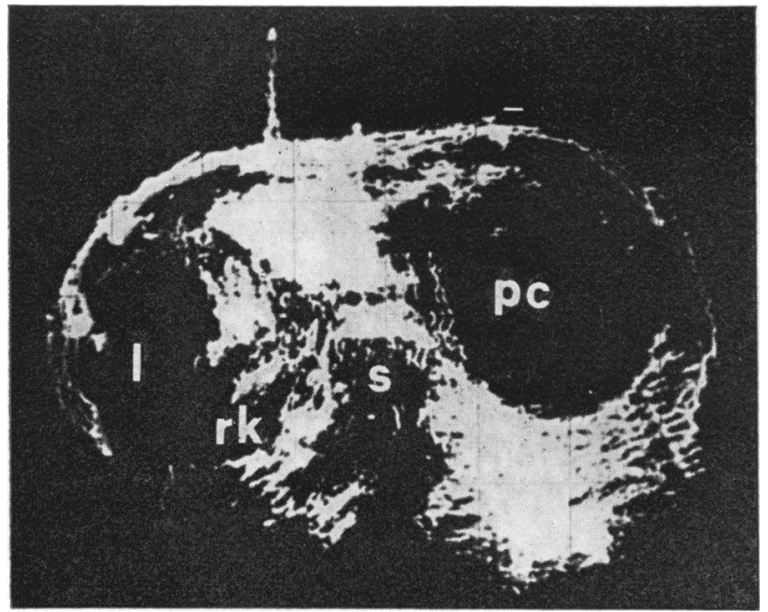

Fig. 5 Pseudocyst of the tail of the pancreas in chronic calcifying pancreatitis. Transverse section $5 \mathrm{~cm}$ from the xiphoid process. A large roundish transonic zone with sharp regular contours can be seen filling almost the entire left portion of the abdomen indicating the psudocyst $(\mathrm{pc})$.

chondrium two months after a severe blow to the abdomen. Echography revealed a roundish cyst about $10 \mathrm{~cm}$ in diameter in the left hypochondrium. It was clearly visible on both longitudinal and transverse scans. There was a distinct area of separation between the cyst formation and the left kidney, which appeared to be of normal shape and size, excluding renal involvement. Surgery confirmed the presence of a cyst in the tail of the pancreas.

\section{CARCINOMA OF PANCREAS}

In all cases examined, echography revealed enlargement of the head of the pancreas, irregular contours, and fairly homogeneous structure not unlike that observed in some cases of chronic pancreatitis (Fig. 7). In two cases scattered multiple echoes were observed in the liver indicating metastases (Fig. 8). Except for the latter two cases, only at surgery was the neoplastic nature of the disease firmly established.

\section{Discussion}

Echography does not often provide a distinct picture of the normal pancreas but is efficient in detecting enlargement of the pancreas (Filly and Freimanis, 1970; Rettenmaier and Gail, 1971; Weill et al., 1971; Stuber et al., 1972).

In our experience, the anteroposterior diameter must be more than $2-3 \mathrm{~cm}$ for the head, and more than $1 \cdot 5-2.5 \mathrm{~cm}$ for the body for echography to be really valid in the diagnosis of pathological states. In our opinion, a diseased pancreas of normal dimensions cannot be detected. None of the normal subjects showed signs of a diseased pancreas according to these criteria.

In this study, we have dealt mainly with chronic pancreatitis and in $65.7 \%$ of cases echography visualized alterations indicative of a pathological date. Apart from the seven cases with larger quantities of gas in the gastrointestinal tract which made recording impossible, in 12 cases echography was in fact negative. In six of these cases, who later underwent surgery, the size of the pancreas was found to

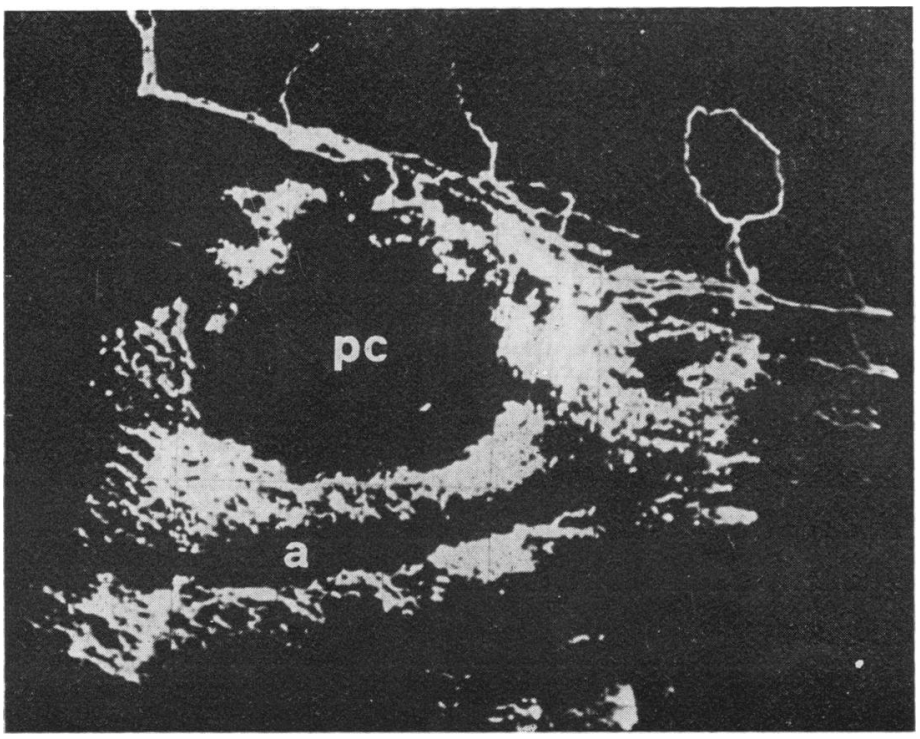

Fig. 6 Pseudocyst formation after acute pancreatitis. Longitudinal section $1 \mathrm{~cm}$ left of the midline. The pseudocyst is clearly visible anterior to the aorta. 


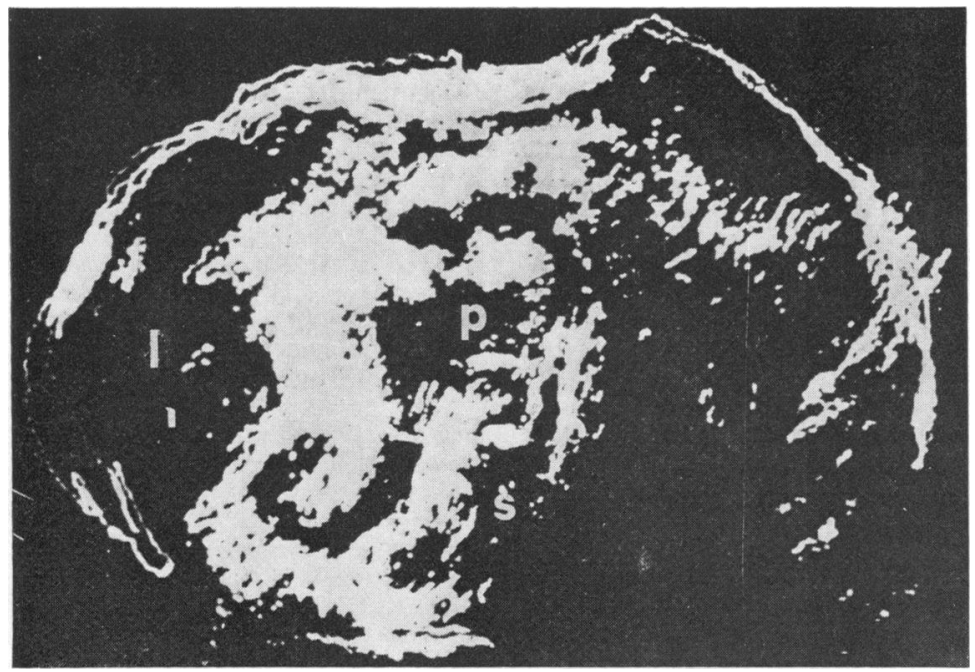

Fig. 7 Carcinoma of the head of the pancreas. Transverse section $6 \mathrm{~cm}$ from the xiphoid process. A homogeneous area with irregular contours, clearly delineated by the surrounding tissue, is visible anterior to the right kidney and vertebral column indicating enlargement of the head of the pancreas.

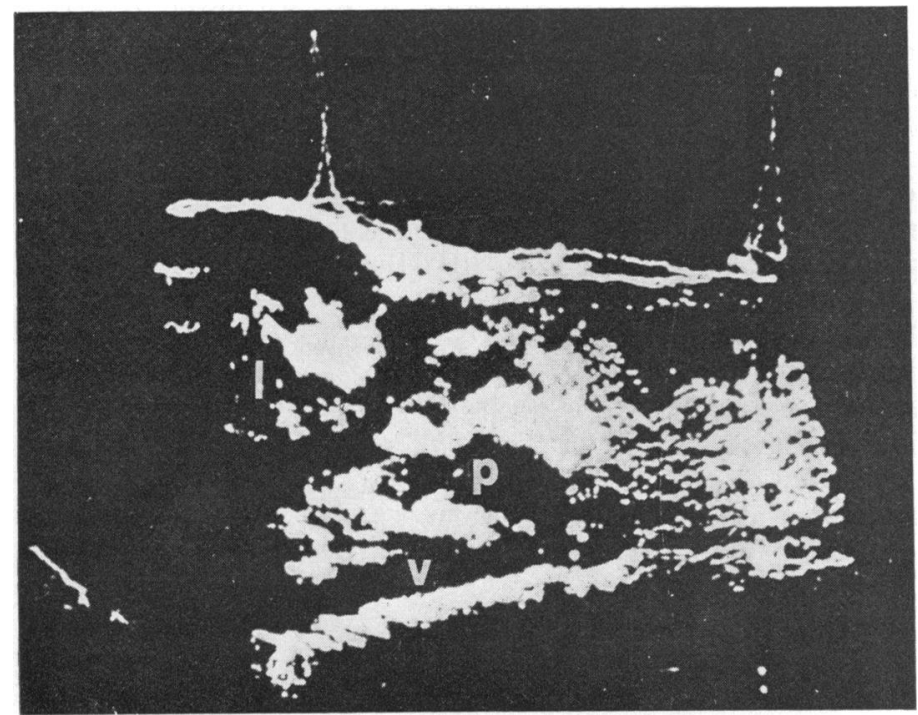

Fig. 8 Carcinoma of the head of the pancreas. Longitudinal section $1 \mathrm{~cm}$ right of the midline. In the angle between the liver and the vena cava an area with the same characteristics as described in the previous figure can be seen. Scattered multiple echoes can be observed in the liver indicating the presence of metastases. $\mathrm{v}=$ vena cava.

be almost normal. In all cases of carcinoma of the pancreas, alterations were visualized on echography which indicated a pathological state.

In spite of the numerous suggestions put forward by some authors for the differentiation of these two diseases (Rettenmaier, 1975; Weill et al., 1975), in neither were we able to diagnose the nature of the illness with certainty. The presence of metastases in the liver can give a good indication as to the type of lesion but an unequivocal diagnosis can be reached only by performing ultrasonically guided percutaneous aspiration biopsy of the pancreas (Smith $e t$ al., 1974; Hancke and Heje, 1975; Rettenmaier, 1975). On the other hand, echography gave unequivocal information of precise diagnostic value in all cases of pancreatic cysts.

Weill et al. (1973) reported that in their experience the percentage of confirmed echographic diagnoses was $94.1 \%$ for chronic pancreatitis, $83.3 \%$ for carcinoma of the pancreas, and $100 \%$ for cysts. The lower percentage reported by Weill et al. for neoplasia is due to the fact that they encountered cases with carcinoma in the body and tail of the pancreas, zones which are much harder to investi- 
gate. In our study carcinoma was confined to the head of the pancreas in all cases. The lower success rate obtained by us in chronic pancreatitis may be due to the fact that many of our patients were examined at a relatively early stage of the disease. In fact, considering the cases showing radiological changes of the duodenum, choledochus or calcification, generally found in the more advanced stages of the disease, and in those in which the head is mainly affected, the percentage of positive echographic results rises to $91 \cdot 3 \%$.

In most cases, echography gave information of greater diagnostic value than either duodenography or cholangiography. Scintillography, however, gave a much higher percentage of positive results, but it should be borne in mind that this technique gives a high percentage of false positives (Liewendahl and Kvist, 1970) and is less precise in the diagnosis of cysts.

In conclusion, we consider echography to be a valuable technique in the diagnosis of pancreatic cysts, even those of quite small dimensions. It can yield useful, if not pathognomonic, information in cancer, particularly if the head of the pancreas is affected. In chronic pancreatitis the results are not diagnostically conclusive but make a valid contribution in conjunction with biological, radiological, and clinical data. In addition, it furnishes a more complete picture of the disease and gives a quantitative estimate of enlargement of the gland. The noninvasive character of echography should be stressed and there are no contraindications to its use. In addition, serial studies may be safely carried out over a period of time to observe the evolution of the disease or the response to therapy. In the next few years it should become one of the standard techniques in the study of pancreatic disease.

\section{References}

Engelhardt, G., and Blauenstein, U. W. (1970). Ultrasound in the diagnosis of pancreatic tumours. Gut, 11, 443-449.

Filly, R. A., and Freimanis, A. K. (1970). Echographic diagnosis of pancreatic lesions. Radiology, 96, 575-582.

Hancke, S., and Heje, C. (1975). The diagnosis of pancreatic lesions by means of ultrasonic scanning and ultrasonically guided puncture. Abstracts of the 2nd European Congress on Ultrasonics in Medicine, Munich, May 1975, p. 43. Junge \& Sohn: Erlangen.

Leopold, G. R. (1972). Pancreatic echography: a new dimension in the diagnosis of pseudocyst. Radiology, 104, 365-369.

Liewendahl, K., and Kvist, G. (1970). Evaluation of pancreatic scanning. Acta Medica Scandinavica, 188, 75-80.

Rettenmaier, G. (1975). Sonography of the pancreastechnique of examinations and results. Abstracts of the 2nd European Congress on Ultrasonics in Medicine, Munich, May 1975, p. 42. Junge \& Sohn: Erlangen.

Rettenmaier, G., and Gail, K. (1971). Echographie pancréatique. Journal de Radiologie d'Electrologie et de Medicine Nucléaire, 53, 745-747.

Smith, E. H., Bartrum, R. J., and Chang, Y. C. (1974). Ultrasonically guided percutaneous aspiration biopsy of the pancreas. Radiology, 112, 737-738.

Stuber, J. L., Templeton, A. W., and Bishop, K. (1972). Sonographic diagnosis of pancreatic lesions. American Journal of Roentgenology, Radium Therapy and Nuclear Medicine, 116, 406-412.

Templeton, A. W., and Stuber, J. L. (1971). Abdominal and retroperitoneal sonography. American Journal of Roentgeneology, Radium Therapy and Nuclear Medicine, 113, 741-748.

Weill, F., Becker, J., Kraehenbuhl, J., Ricatte, J. P., Kasozi, H., and Prevotat, N. (1971). Apport de la tomoéchographie dans l'exploration pancréatique. Annales de Radiologie, 14, 735-746.

Weill, F., Bourgoin, A., and Eisencher, A. (1975). Ultrasonic diagnosis between chronic pancreatitis and carcinoma. Abstracts of the 2nd European Congress on Ultrasonics in Medicine, Munich, May 1975, p. 43. Junge \& Sohn: Erlangen.

Weill, F., Kraehenbuhl, J., Becker, J. C., Bourgoin, A. and Gillet, M. (1973). Fiabilité de l'exploration echotomographique du pancréas. Nouvelle Presse Medicale, 2, 2127. 